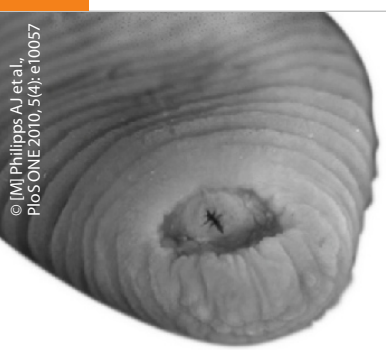

Halten Sie sich fest: T. rex ist zurückgekehrt! Zwar in einer etwas anderen Größendimension - aber nicht minder furchterregend! Mit mächtigen Zähnen beißt sich das tückische Tierchen in der Nase fest. Sein Lebenselixier: Blut!

Das Tier besitzt nur einen Kiefer, der mit riesigen Zähnen besetzt ist. Riesig ist dabei relativ, misst der Wurm selbst

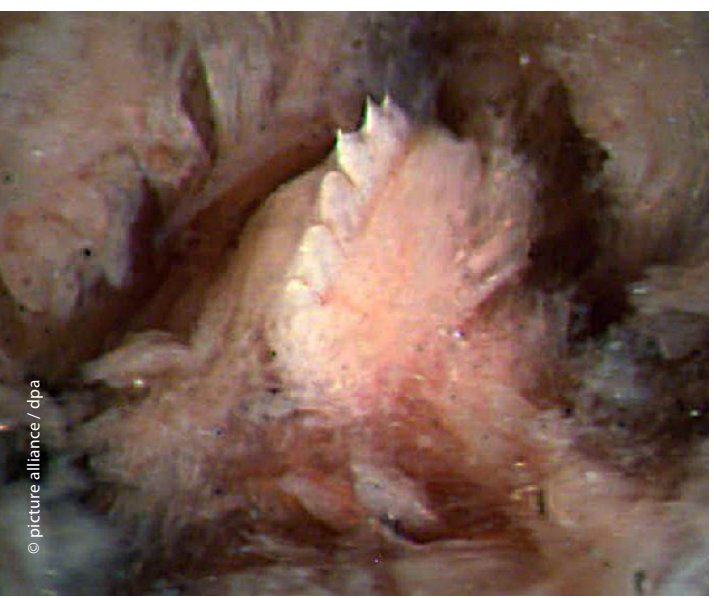

Perfekte Zapfanlage: Mächtige Zähne trägt der Blutegel T. rex (kl. Bild oben) auf seinem einen Kiefer. doch nur $4 \mathrm{~cm}$ bei einem Durchmesser von $0,95 \mathrm{~cm}$.

Gefunden wurde dieser bis zu $7 \mathrm{~cm}$ lange Blutegel namens Tyrannobdella rex in den Nasen von drei peruanischen Kindern nach dem Baden in Seen oder Flüssen. Bis auf die erschreckend großen Zähne hat der neue T. rex aber nichts mit seinem verstorbenen Namensvetter gemein. Doch auch er bringt die Forscher zum Staunen: „Es ist keine andere Egelart bekannt, die nur einen Kiefer mit solch großen Zähnen hat", schreiben sie in der Zeitschrift „PLoS ONE“. Statt der Haut wie seine uns bekannten Verwandten bevorzugt T. rex die Nasenschleimhaut - meist von Tieren, aber auch Menschen können betroffen sein, wie die beschriebenen Fälle zeigen.

Dort verursachten sie frontale Kopfschmerzen, aber keine Blutungen oder respiratorische Probleme. Eines der Kinder, ein 9-jähriges Mädchen, hatte zudem ein glitschiges Gefühl in der Nase. Ihre Eltern entdeckten, dass sich in ihrem rechten Nasenloch ein kleiner schwarzer Wurm bewegte - und hatten somit einen leibhaftigen T. rex gesehen! (sko) Phillips AJ et al., PLoS ONE 2010, 5(4):e10057

\section{Suchtgefahr: Solarium macht abhängig!}

Bei gut einem Drittel der Solariumgänger entspricht der Drang nach der schnellen Bräune den Kriterien einer Sucht, so das Ergebnis einer aktuellen Studie. Mit zwei modifizierten Fragebögen, die aus dem Sucht-Screening stammen, untersuchten US-Forscher 421 Studenten einer amerikanischen Universität auf ihr Bräunungsverhalten. 229 von ihnen gaben an, die Sonnenbank regelmäßig zu drücken. Bei über 30\% dieser Probanden ergab die Auswertung ausreichend Kriterien, um eine Substanzabhängigkeit zu erfüllen. Mosher CA et al, Arch Dermatol 2010, 146:412

\section{Prostatakrebs-Risiko einfach berechnen}

Je mehr direkte Verwandte eines Mannes von Prostatakrebs betroffen sind, desto höher ist sein persönliches Risiko, ebenfalls an dem malignen Tumor zu erkranken. In der bisher größten Studie zu familiärem Prostatakrebs mit insgesamt 26.651 Teilnehmern errechneten Forscher z. B. ein 23-mal höheres Erkrankungsrisiko für Männer mit drei betroffenen Brüdern als in der Kontrollgruppe mit gesunden Angehörigen. Je jünger die Verwandten dabei waren, desto mehr stieg die Gefahr für den Einzelnen. Brandt A et al, Eur Urol 2010 (online first)

\section{CME hilft Ihnen, Ihr Punktekonto zu füllen!}

Mit unseren drei zertifizierten Fortbildungen können Sie pro Heft auf CME.springer.de/CME kostenlos 9 Punkte sammeln. Und so geht's:

\section{Kostenlos teilnehmen in vier Schritten}

\section{Auswählen}

Wählen Sie online auf CME.springer. de/CME den Link zum aktuellen Heft aus. Entscheiden Sie nun, welche Fortbildungseinheiten Sie zum Erwerb von CME-Punkten nutzen wollen. Die Teilnahme an den drei Einheiten der aktuellen Ausgabe ist kostenlos.

\section{Registrieren/Anmelden}

Sind Sie bereits bei CME.springer.de registriert? Dann genügt zur Anmeldung die Angabe Ihrer persönlichen Zugangsdaten. Falls Sie zum ersten Mal teilnehmen, bitten wir Sie, sich einmalig zu registrieren. Wir senden Ihnen dann per E-Mail Ihre persönlichen Zugangsdaten zu. Bitte benutzen Sie diese für alle weiteren Teilnahmen zur Anmeldung (Login).

\section{Teilnehmen}

Sie können die elektronische Version der Fortbildung online lesen, ausdrucken oder direkt die 10 CME-Fragen beantworten. Die kostenlose Teilnahme ist 3 Monate nach Erscheinen der Zeitschrift möglich.

\section{CME-Punkte sammeln}

Nach erfolgreicher Beantwortung von 7 der 10 CME-Fragen senden wir Ihnen Ihre Teilnahmebestätigung samt Punkten umgehend per E-Mail zu. Diese können Sie bei Ihrer zuständigen Landesärztekammer einreichen. 